\section{Jäger und Sammler}

30.06.2009: 95\% der deutschen Ärzte hatten zum Stichtag der ersten Fünfjahresfrist die 250 CME-Punkte eingereicht. 4 Quartale lang konnten Punkte noch nachreicht werden.

- ca. 1000 Ärzten drohte am Ende noch der Zulassungsentzug.

99\% der 114.000 Vertragsärzte waren gesichert! - die Einführung der Fortbildungspflicht verlief „völlig unproblematisch", resümierte die BÄK 2009.

- Weit über $\mathbf{3 0 0 . 0 0 0}$ Fortbildungsveranstaltungen werden laut BÄK pro Jahr geprüft und anerkannt.

- Mit CME sammeln Sie mit jeder Ausgabe bis zu 9 CME-Punkte - diesmal sogar bis zu 27 CME-Punkte!

Ärzte Zeitung, 09.12.2009/04.07.2011; Pressestelle BÄK

\section{Punkte}

\section{für Tauchunfälle}

Bei Berufstauchern, aber auch aufgrund der hohen Beliebtheit des Tauchens als Freizeitaktivität, kommt es immer wieder zu Tauchunfällen. Von notfallmedizinischer Bedeutung sind hier besonders die sogenannten Dekompressionsunfälle. Ertrinkungsunfälle wiederum stellen international vor allem bei jüngeren Menschen in den Todesursachenstatistiken einen erheblichen Anteil dar. Erfahren Sie mehr zur Pathophysiologie des Tauchunfalls und erlernen Sie die leitliniengerechte Therapie von Tauchunfällen sowie die Therapiemaßnahmen nach einem Ertrinkungsunfall - die Fortbildung „Tauchund Ertrinkungsunfälle“ finden Sie in diesem Heft ab Seite 61.

\title{
Neues e.Tutorial plus auf springermedizin.de
}

Erleben Sie Fortbildung neu! Mit dem e.Tutorial plus „Notfall: Akuter Thoraxschmerz und akutes Koronarsyndrom“ können Sie auf springermedizin.de multimedial, interaktiv und zertifiziert punkten. Sie lernen Ursachen und Differenzialdiagnosen des akuten Thoraxschmerzes kennen und können anschließend mit Scoring-Systemen das Risiko einer lebensbedrohlichen Erkrankung einschätzen. Nach Absolvieren der Fortbildung verstehen Sie Symptomatik und Risikoeinschätzung des akuten Koronarsyndroms und kennen die
Empfehlungen zur präklinischen SofortTherapie und den Behandlungsalgorithmus. Der Online-Kurs unterstützt Sie mit interaktiven Lerneinheiten, die Ihr individuelles Lerntempo berücksichtigen. Dabei vermitteln praxisnahe Kasuistiken das richtige Vorgehen in der Praxis und EKGBilderstrecken verdeutlichen relevante klinische Fälle. Zum Schluss festigen interaktive Wissensfragen das erlernte Wissen. Neugierig? Dann loggen Sie sich unter springermedizin.de/eAkademie ein und wählen den entsprechenden Kurs aus. (red)

Schneller als die Post erlaubt

\section{Mit e.Med die Ipad-Edition der Ärzte Zeitung lesen!}

Wer das e.Med-Paket von Springer Medizin bucht, bekommt immer wieder mehr geboten: Zusätzlich zu den Inhalten von über 600 medizinischen Fachzeitschriften, über 500 zertifizierte Fortbildungen, detaillierter Kongressbericht- erstattung, Experteninterviews und täglich aktuellen Nachrichten aus der Welt der Medizin und der Gesundheitspolitik gibt es nun auch die als „Bestes Mobiles Angebot 2013“ ausgezeichnete exklusive Ipad-Edition der Ärztezeitung im Paket! Und all das zu einem monatlichen festen Pauschalpreis von 32,25 EUR. Ob erstmal 30 Tage lang das Angebot gratis testen oder gleich buchen - hier ist der Weg zu Ihrem e.Med:

www.springermedizin.de/eMed

\section{Affenstark: Punkten Sie kostenfrei!}

Sommer, Sonne, Punktezeit: Mit der Sommerakademie ermöglichen wir es Ihnen auch dieses Jahr wieder, in schöner Tradition kostenfrei 27 CME-Punkte zu sammeln. Bis Ende August können Sie sich dazu auf springermedizin.de registrieren lassen und mittels der Fortbildungsidentifikationsnummer (FIN) direkt aus dem Vollen schöpfen: $\mathrm{Ob}$ Morbus Crohn, Rückenschmerz oder Diabetes \& Co. - die Fortbildung ist garantiert!

Claudia Daniels Redakteurin

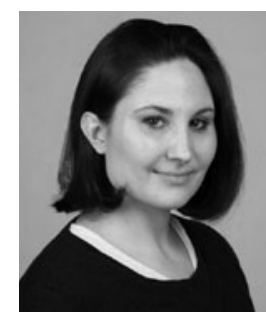

Springer Medizin e.Akademie Ihre Top-Ten-Fortbildungen 2013

Diese CME-Beiträge aus dem Jahr 2013 wurden in der Springer e.Akademie am häufigsten absolviert:

- Sepsis - Wissen für die Praxis

- Vorhofflimmern - Aktuelle Empfehlungen zur Diagnostik und Behandlung

- Hyperthyreose

- Diagnose \& Therapie der diastolischen Herzinsuffizienz

- Osteoporose-Therapie

- Akuter Asthmaanfall und exazerbierte COPD

- Update Typ-1-Diabetes

- Akutversorgung des ischämischen Schlaganfalls

- Differenzialdiagnose und Behandlung des akuten Kopfschmerzes

- Effektiver Sonnenschutz für jeden Hauttyp

Fehlt Ihnen noch ein Top-Ten-Beitrag? Die Top-Ten-Fortbildungen finden Sie, indem Sie den Titel in die Suche auf - www.springermedizin.de/ eAkademie eingeben. 